\title{
Theory of Dipole-Exchange Spin Excitations in a Spherical Ferromagnetic Nanoshell, consideration of the Boundary Conditions
}

\author{
V.V. Kulish \\ Department of general and experimental physics, National Technical University of Ukraine "Igor Sikorsky Kyiv Polytechnic \\ Institute", 37 Peremogy prosp., 03056, Kyiv, Ukraine
}

\begin{abstract}
The paper continues study of dipole-exchange spin excitations in a spherical ferromagnetic nanoshell started by the author in the previous paper. The proposed model considers the magnetic dipole-dipole interaction, the exchange interaction, the anisotropy effects and the damping effects. A new method of obtaining the values' spectrum of the wavenumbers for the investigated excitations - the method based on the application of general boundary conditions - is proposed. Consequently, the values' spectra of the wavenumbers and the frequencies of the investigated excitations are obtained in addition to the previously obtained dispersion law. Exploitation of the above-mentioned method essentially extends the area of application of the obtained results compared to the previous paper. The obtained dependence of the wavenumber on the angular mode number is shown to be weak and close to linear. On the other hand, the obtained dependence of the wavenumber on the radial mode number is shown to be essential. The obtained spectrum of wavenumbers' values is shown to transform to a quasi-one-dimensional form once specific conditions are satisfied; these conditions are found.
\end{abstract}

Keywords- Magnetic dynamics, Spin excitation, Dipole-exchange theory, Ferromagnetic nanosystem, Spherical nanoshell.

\section{INTRODUCTION}

Nowadays, a rapidly developing sub-field of information technologies is dedicated to creating data storage, transfer and processing devices based on the applications of spin waves in nanosystems [1-3]. One of the key problems for developing such technologies is theoretical modeling of spin-wave processes in these nanosystems. Such modeling is required not only for direct applications of spin waves, but also for synthesizing materials with preset magnetic properties as these properties are often influenced by spin-wave processes. This modeling, in turn, requires deeper understanding of the corresponding processes in magnetic nanosystems. In the proposed paper, one of the problems of the above-described type is solved.

As it has been shown by numerous studies (see, e.g., the review [4]), properties of nanosystems - in particular, spin-wave properties - depend essentially on their size and shape. Unfortunately, a general theory of spin waves in magnetic nanosystems has not been created at the moment. Therefore, spin waves in nanosystems of different geometries are studied separately. Among the variety of magnetic nanosystems of different configurations, a special class is represented by shelltype ferromagnetic nanosystems (nanoshells, nanotubes and others). These nanosystems exhibit unique - not inherent to traditional continuous nanosystems - properties that are prospective for numerous technical applications. In particular, magnetic properties of such nanosystems can be regulated more flexibly than properties of corresponding continuous nanosystems [5]. However, such nanosystems remain poorly researched at the moment. In particular, study of spin-wave processes in synthesized recently ferromagnetic nanoshells [5-7] represent and actual topic of research.

The paper continues the study of dipole-exchange spin excitations in a spherical ferromagnetic nanoshell started by the author in the paper [8]. The magnetic dipole-dipole interaction, the exchange interaction, the anisotropy effects and the damping effects are considered. In the previous paper of the author [8], a dispersion relation for the above-described spin excitations has been obtained. However, for complete description of such excitations, this relation should be complemented by a values' spectrum of the wavenumber (in particular, this allows to obtain the spectrum of values of the spin excitations' frequencies). For the most nanosystems, that represents more challenging task than just finding the dispersion relation. In the paper [8], the above-mentioned spectrum has been obtained only for a very specific particular case (the material outside the nanoshell has been assumed to be a high-conductivity metal) thus essentially limiting the area of application of the entire obtained result. The proposed paper overcomes this limitation by applying a different - essentially more general - method of obtaining the above-mentioned spectrum. As a result, the values' spectrum of the spin excitation wavenumbers and 
frequencies - that can be applied for much wider range of nanoshell configurations - are obtained for an essentially more general case of the nanoshell configuration. The obtained spectrum of wavenumbers is shown to transform to a quasi-onedimensional form once specific conditions are satisfied; these conditions are found.

\section{Problem STATEMENT: Model Description}

Let us consider a spherical ferromagnetic nanoshell composed of a uniaxial ferromagnet of the "easy axis" type. Let us denote the ferromagnet parameters as follows: the exchange constant $\alpha$, the uniaxial anisotropy parameter $\beta$, the gyromagnetic ratio $\gamma$, the ground state magnetization $\vec{M}_{0}$ (is considered constant in absolute value inside the shell), the dissipation parameter $\alpha_{G}$ (the Hilbert term is used for consideration of the dissipation). We assume that the external magnetic field is absent, and the easy magnetization axis - and, therefore, the ground state magnetization - is directed radially inside the nanoshell. Let us denote the internal shell radius $a$ and the external radius $b$ (see Fig.1.).



FIGURE 1. The spherical nanoshell that is studied in the paper.

Let us consider a spin wave (spin excitation in a form of standing wave, to be exact) propagating in the nanoshell so that the magnetization $\vec{m}$ and the magnetic field $\vec{h}$ of the wave are small perturbations of the overall magnetization $\vec{M}$ and the magnetic field inside the ferromagnet $\vec{H}^{(i)}$, correspondingly (linear wave). Thus, the relations $|\vec{m}|<<\left|\vec{M}_{0}\right|,|\vec{h}|<<\left|\vec{H}_{0}^{(i)}\right|$ fulfill, where $\vec{H}_{0}^{(i)}$ is the ground state internal magnetic field (so that $\vec{M}=\vec{M}_{0}+\vec{m}, \vec{H}^{(i)}=\vec{H}_{0}^{(i)}+\vec{h}$ ). Let us find the dispersion relation and the wavenumber values' spectrum for such linear spin excitations.

For the investigated spin excitation, let us use the magnetostatic approximation, assuming that the magnetic potential $\Phi$ exists and, therefore, $\vec{h}=-\nabla \Phi$. After introducing amplitudes $\vec{m}_{0}, \vec{h}_{0}$ for the magnetization and the magnetic field perturbations, correspondingly (so that $\vec{m}(\vec{r}, t)=\vec{m}_{0}(\vec{r}) \exp (i \omega t), \vec{h}(\vec{r}, t)=\vec{h}_{0}(\vec{r}) \exp (i \omega t)$, where $\omega$ is the wave frequency), we can write down the following relations for the magnetic potential: $\vec{h}_{0}=-\nabla \Phi_{0}, \Phi=\Phi_{0} \exp (i \omega t)$. The outside material is considered non-magnetic so the relations $\vec{m}_{0}=0, \Delta \Phi_{0}=0$ fulfill outside the investigated ferromagnet. After combining the linearized Landau-Lishitz equation with the Maxwell equation $\operatorname{div} \vec{H}^{(i)}=-4 \pi \operatorname{div} \vec{M}$ and eliminating the magnetization amplitude in the resulting system of equations, the following equation for the magnetic potential of the investigated waves can be obtained:

$$
\left(\frac{\omega^{2}}{\gamma^{2} M_{0}^{2}}-\left(\tilde{\beta}-i \frac{\alpha_{G} \omega}{\gamma M_{0}}-\alpha \Delta\right)\left(\tilde{\beta}-i \frac{\alpha_{G} \omega}{\gamma M_{0}}+4 \pi-\alpha \Delta\right)\right) \Delta \Phi_{0}+4 \pi\left(\tilde{\beta}-i \frac{\alpha_{G} \omega}{\gamma M_{0}}-\alpha \Delta\right) \frac{1}{r^{2}} \frac{\partial}{\partial r}\left(r^{2} \frac{\partial \Phi_{0}}{\partial r}\right)=0
$$

(see [8]), here the spherical coordinates $(r, \theta, \varphi)$ are used and the value $\widetilde{\beta}=\beta+H_{0}^{(i)} / M_{0}$.

In order to obtain the values' spectrum of wavenumbers of the investigated spin excitation, let us use the boundary conditions for the magnetic field. Assuming that standard boundary conditions fulfill for the ground state magnetization and the magnetic field, we can write down $b_{1 n}=b_{2 n}, h_{1 \tau}=h_{2 \tau}$ on the boundary of the considered ferromagnet (here medium 1 is the ferromagnet, medium 2 is the external medium, $n$ means normal and $\tau$-tangential to the boundary vector component, $\vec{b}$ is the magnetic induction vector of the wave). For the vectors $\vec{h}, \vec{m}$ we obtain $h_{1 n}-h_{2 n}=4 \pi m_{n}, h_{1 \tau}=h_{2 \tau}$ (as the outside 
environment is non-magnetic). For the magnetic potential, these conditions together with the condition of the potential continuity on the ferromagnet boundary can be written in the following form:

$$
\left\{\begin{array}{c}
\left.\Phi_{0}\right|_{1}=\left.\Phi_{0}\right|_{2} \\
\left(\nabla \Phi_{0}\right)_{1 \tau}=\left(\nabla \Phi_{0}\right)_{2 \tau} \\
\left.\frac{\partial \Phi_{0}}{\partial n}\right|_{1}-\left.\frac{\partial \Phi_{0}}{\partial n}\right|_{2}=4 \pi m_{0 n}
\end{array}\right.
$$

Note that the conditions (2) contains not only the magnetic potential, but also a normal component of the magnetization of the wave, so for the complete solution of the problem, generally speaking, the above-mentioned magnetization should be found. For the investigated nanosystem, however, the ground state magnetization is directed radially. As the spin wave magnetization $\vec{m}_{0}$ is normal to this direction, the component $m_{0 n}$ on the nanoshell boundary vanishes: $m_{0 n}=0$. Therefore, the last condition in (2) can be rewritten as follows:

$$
\left.\frac{\partial \Phi_{0}}{\partial n}\right|_{1}-\left.\frac{\partial \Phi_{0}}{\partial n}\right|_{2}=0
$$

In other nanosystem configuratons such simplification can be made, for instance, by the means of imposing fixed boundary conditions for the magnetization.

The system (2) together with the boundary conditions (2), (3) will be used as starting relations during the investigation of the above-described spin excitations.

\section{SPECTRAL CHARACTERISTICS OF THE SPIN ESCITATIONS}

An approximate solution of the equation for the magnetic potential (1) can be written in the form $\Phi_{0}(r, \theta, \varphi)=\left(A_{1} j_{l}(k r)+A_{2} n_{l}(k r)\right) Y_{l m}(\theta, \varphi)$, here $l$ and $m$ are integers $(-l \leq m \leq l)$, numbers of the angular excitation mode, $j_{l}$ and $n_{l}$ are the spherical Bessel and Neumann functions of the order $l$, correspondingly, $Y_{l m}$ are the spherical polynomials, $k$ is the radial wavenumber and $A_{1}, A_{2}$ are constants. This form of solution can be used when the expression $l(l+1) / r^{2}$ can be considered approximately constant inside the shell or small compared to $k^{2}$. The first condition is satisfied, in particular, when the shell is thin, so the relation $\left(b^{2}-a^{2}\right) / a^{2}<<1$ fulfills. The second condition is satisfied, in particular, in the case of short waves $(k a>>l)$ and in the case of purely radial spin excitations $(l=0)$, see [8]. In the case of thin nanoshell $\left(b^{2}-a^{2}\right) / a^{2}<<1$ after substituting the given above solution into the equation (4) the following approximate dispersion relation can be obtained:

$$
\omega=\frac{|\gamma| M_{0}}{1+\alpha_{G}^{2}}\left(\sqrt{\left(1+\alpha_{G}^{2}\right)\left(\alpha^{2} k^{4}+2 \alpha \tilde{\beta} k^{2}+\tilde{\beta}^{2}+4 \pi \alpha\left(1+\frac{\tilde{\beta}}{\alpha k^{2}}\right) \frac{l(l+1)}{r_{0}^{2}}\right)-\alpha_{G}^{2}\left(\frac{K}{k}\right)^{4}}-i \alpha_{G}\left(\frac{K}{k}\right)^{2}\right)
$$

here $K^{2}(k)=\alpha k^{4}+\tilde{\beta} k^{2}+2 \pi l(l+1) / r_{0}^{2}, r_{0}=\sqrt{\left(b^{2}+a^{2}\right) / 2}$ (see [8]). For a spin wave that can practically be excited, the dissipation parameter $\alpha_{G}$ should be less or of an order of 0.1 so that $\alpha_{G}^{2}<<1$ and, therefore, everywhere in (4) the replacement $1+\alpha_{G}^{2} \rightarrow 1$ can be made. After that, the dispersion relation (4) can be rewritten as follows:

$$
\omega=|\gamma| M_{0}\left(\sqrt{\alpha^{2} k^{4}+2 \alpha \tilde{\beta} k^{2}+\tilde{\beta}^{2}+8 \pi \alpha\left(1+\frac{\tilde{\beta}}{\alpha k^{2}}\right) \frac{l(l+1)}{b^{2}+a^{2}}}-i \alpha_{G}\left(\alpha k^{2}+\tilde{\beta}+\frac{4 \pi l(l+1)}{k^{2}\left(b^{2}+a^{2}\right)}\right)\right)
$$

It was mentioned in the Introduction that in order to complete the presented theoretical analysis, the spectrum of values of the radial wavenumber also needs to be found. In the previous paper of the author [8], the above-mentioned spectrum has been obtained only for a very specific particular case (the material outside the nanoshell has been assumed to be a highconductivity metal) thus essentially limiting the area of application of the entire obtained result. On the other hand, using 
consideration described in the Section 2 of the current paper allows obtaining the specified spectrum in a very general case. The area of application of the result will be limited only by the nanoshell model described in the Section 2.

The absence of the radial excitations $(k=0)$ for the investigated nanoshell corresponds to the spatially uniform spin oscillations (ferromagnetic resonance). In particular, this takes place when the shell thickness is less than the exchange length so radial modes of excitations are not possible. When the radial excitations are present $(k \neq 0)$, the magnetic potential outside the nanoshell can be sought in the form $\Phi_{0}^{e}(r, \theta, \varphi)=F^{e}(r) Y_{l m}(\theta, \varphi)$, where the function $F^{e}$ satisfies the following equation:

$$
F^{e \prime \prime}+\frac{2}{r} F^{e \prime}-\frac{l(l+1)}{r^{2}} F^{e}=0
$$

Solution of this equation that satisfies the continuity condition on the nanoshell boundary and is limited when $r \rightarrow \infty$ as well as in the point $r=0$ can be written in the following form:

$$
F^{e}=\left[\begin{array}{cc}
\left(B_{1} j_{l}(k a)+B_{2} n_{l}(k a)\right)(r / a)^{l}, & r \leq a \\
\left(C_{1} j_{l}(k b)+C_{2} n_{l}(k b)\right)(r / b)^{-(l+1)}, & r>b
\end{array}\right.
$$

here $B_{1}, B_{2}, C_{1}, C_{2}$, are constants. The corresponding magnetic potential satisfies the Laplace equation and both the first and the second conditions in (2). Finaly, from the condition (3) the sought expression for the wavenumber can be found. This expression is implicit and can be written as follows:

$$
\frac{k j_{l}{ }^{\prime}(k b)+((l+1) / b) j_{l}(k b)}{-k n_{l}{ }^{\prime}(k b)-((l+1) / b) n_{l}(k b)}=\frac{k j_{l}{ }^{\prime}(k a)-(l / a) j_{l}(k a)}{-k n_{l}{ }^{\prime}(k a)+(l / a) n_{l}(k a)}
$$

Let us take advantage of the fact that the investigated nanoshell is thin: $(b-a) / a<<1$ and, therefore, $k a>>1, k b>>1$ for typical nanoshells. This allows simplifying the obtained spectrum by means of applying the asymptotics of the spherical Bessel and Neumann functions. For $\cos (k a) \neq 0, \cos (k b) \neq 0(8)$ can be rewritten as follows:

$$
\operatorname{tg}(k(b-a))=\frac{k((l+1) b+l a)}{k^{2} a b-l(l+1)}
$$

If the additional condition $k^{2} a b>>l(l+1)$ fulfills, the right hand side in the last relation can be neglected $(\operatorname{tg}(k(b-a))=0)$ and, therefore, the spectrum for the radial wavenumber has an explicit - quasi-one-dimensional - form:

$$
k=\frac{\pi n}{b-a}
$$

where $n$ is an integer, the number of the radial excitations mode. A spectrum of such form can be expected for effectively one-dimensional nanosystem, e.g., a flat ferromagnetic film. Therefore, in the particular case when $k^{2} a b>>l(l+1)$ (so the angular excitations are neglected), the frequencies' spectrum of the considered spin excitations can be written as follows:

$$
\omega=|\gamma| M_{0}\left(\sqrt{\alpha^{2}\left(\frac{\pi n}{b-a}\right)^{4}+2 \alpha \tilde{\beta}\left(\frac{\pi n}{b-a}\right)^{2}+\tilde{\beta}^{2}+8 \pi \alpha\left(1+\frac{\tilde{\beta}}{\alpha}\left(\frac{\pi n}{b-a}\right)^{-2}\right) \frac{l(l+1)}{b^{2}+a^{2}}}-i \alpha_{G}\left(\alpha\left(\frac{\pi n}{b-a}\right)^{2}+\tilde{\beta}+\frac{4 l(l+1)(b-a)^{2}}{\pi n^{2}\left(b^{2}+a^{2}\right)}\right)\right)(11)
$$

In a general case - when the condition $k^{2} a b>>l(l+1)$ is not satisfied - this spectrum is defined by the equation (5) with the values of $k$ that satisfy the relation (9). Note that the condition $k^{2} a b>>l(l+1)$ for a set values of $a, b$ corresponds to either large value of $k$ or small value of $l$, depending on which of these values is set.

Analogous considerations can be used for the case of short waves $(k a>>l)$ and in the case of purely radial spin excitations $(l=0)$. Dispersion relation for such cases can be written in the following form: 


$$
\omega=\frac{|\gamma| M_{0}}{1+\alpha_{G}^{2}}\left(\tilde{\beta}+\alpha k^{2}\right)\left(1-i \alpha_{G}\right)
$$

For such cases, the relation $k^{2} a b>>l(l+1)$ always fulfill and, therefore, a quasi-one-dimensional spectrum (10) for the radial wavenumber can be used. After replacing $1+\alpha_{G}^{2} \rightarrow 1$ we obtain the values' spectrum of the frequencies as follows:

$$
\omega=|\gamma| M_{0}\left(\tilde{\beta}+\alpha\left(\frac{\pi n}{b-a}\right)^{2}\right)\left(1-i \alpha_{G}\right)
$$

Let us analyze the obtained relations.

\section{DISCUSSION}

First, let us point out that for the investigated nanoshell the spin excitations' frequencies spectrum becomes essentially discrete (unlike the nanosystems that are nanosized only in two dimensions - nanotubes, nanowires and so on). This fact implies from the obtained relations (5), (9), (10).

Then, let us make numerical estimations of the spin excitations' frequency in the considered nanoshell. Note that the wavenumber $k$ is restricted, on the one hand, by the nanoshell thickness $b$ - $a$ (unities to tens of nanometers for typical nanoshells), and, on the other hand, by the interatomic distance $d_{0}$ (several angstroms for typical materials). Therefore, the wavenumber lies in the interval $10^{3}-10^{5} \mathrm{~m}^{-1}$ and the sought frequency for typical ferromagnet parameters $\left(\beta \sim 1, \alpha \sim 10^{-12} \mathrm{~cm}^{-2}\right.$, $\gamma=10^{7} \mathrm{~Hz} / \mathrm{Gs}, M_{0}=10^{3} \mathrm{Gs}$ ) lies in the interval $10^{10}-10^{12} \mathrm{~Hz}$. That, really, corresponds to the typical spin waves' frequencies. The above-mentioned considerations also limit the numbers of angular and radial modes: the number of possible radial modes for typical nanoshells is $n_{\max } \sim(b-a) / d_{0} \sim 10 \div 100$, and the number of possible angular modes is $l_{\max } \sim 2 \pi r_{0} / d_{0} \sim 50 \div 500$.

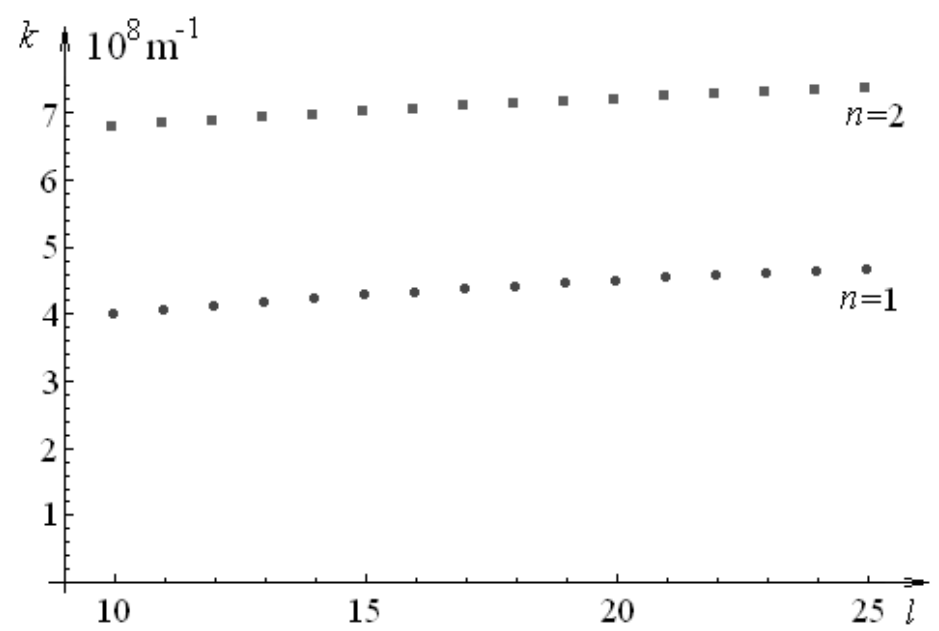

FIGURE 2. The dependence of the radial wavenumber on the angular mode number $l$ for the first two radial modes $(n=1, n=2)$. Round dots (lower set) represents the dependence for $n=1$, square (upper set) - for $n=2$.

Finally, let us analyze the obtained expression for the wavenumbers' spectrum in the case when it is not quasi-onedimensional - the condition $k^{2} a b>>l(l+1)$ does not fulfill, so the relation (9) must be used. Numerical analysis shows that for a given $l$, there exists a number of solutions of (9) that correspond to different radial modes. Let us choose typical values $a=50 \mathrm{~nm}, b=60 \mathrm{~nm}$. Then, the condition $k^{2} a b>>l(l+1)$ does not fulfill starting from $l \sim 10$ and possible values of $k$ start from $k_{\min } \sim \pi /(b-a) \sim 3 \cdot 10^{8} \mathrm{~m}^{-1}$. Numerical analysis shows that for a given $n$, the wavenumber dependence on $l$ is weak, increasing and close to linear (see Fig. 2) until the next branch of $k$ values becomes possible for the given $a, b$. (The last mentioned effect in most cases can be practically neglected - for instance, for the above-mentioned nanoshell sizes such passage takes place when $l \sim 300$ ). On the other hand, the dependence of the wavenumber on the radial mode number is essential. It can be seen from the graph for $n=1$ and $n=2$, and from numerical analysts for higher values of $n$. For instance, for $n=3$ in the same range of $l$ the wavenumber ranges from approximately $9.8 \cdot 10^{8} \mathrm{~m}^{-1}$ to approximately $10.2 \cdot 10^{8} \mathrm{~m}^{-1}$. 


\section{CONCLUSION}

Thus, dipole-exchange spin excitations in a spherical ferromagnetic nanoshell have been investigated in the paper. The magnetic dipole-dipole interaction, the exchange interaction, the anisotropy effects and the damping effects have been considered. The dispersion law for the above-mentioned excitations - obtained in the previous paper of the author - has been complemented with the spectra of wavenumber values and the frequencies' values. The above-mentioned spectra have been obtained using general boundary conditions for the magnetic field. In the previous paper of the author [8], the abovementioned spectra have been obtained only for a very specific particular case (the material outside the nanoshell has been assumed to be a high-conductivity metal) thus essentially limiting the area of application of the entire obtained result. On the other hand, the method proposed in the current paper does not require any additional assumptions. Therefore, the obtained results can be used for any spherical ferromagnetic nanoshell of the studied configuration as long as the general nanoshell model used in the paper (thin nanoshell, linear excitations, constant absolute value of the magnetization vector etc.) can be applied - and the mentioned model is applicable for typical spherical ferromagnetic nanoshells synthesized nowadays.

A graphical representation of the obtained spectrum has been given and the numerical analysis of the obtained results has been performed. The analysis has shown that the dependence of the wavenumber on the angular mode number is weak and close to linear, while the dependence of the wavenumber on the radial mode number is essential. It has also been shown that if the condition of large wavenumber or the condition of small number of an angular mode is satisfied, the obtained spectrum transforms to a quasi-one-dimensional form (analogous to one observed in a flat film).

The method proposed in the paper can be applied to nanoshells of more complex configurations - for instance, a nanorice nanoshell - as well as for more complex configurations of shell-type nanosystems in general. However, one have to bear in mind that for configurations of ground state magnetization different from the one used in the paper, additional conditions (for instance, fixed boundary conditions for the magnetization) should be applied.

\section{ACKNOWLEDGEMENTS}

The author is grateful to Corresponding Member of the Academy of Pedagogical Sciences of Ukraine, Dr.Sci. (Phys.-Math.), Professor Yu.I. Gorobets for his attention to this work, fruitful discussions and valuable remarks.

\section{REFERENCES}

[1] C. Chappert, A. Fert, and F.N. Van Dau, "The emergence of spin electronics in data storage", Nat. Mater., vol.6, No.11, pp. 813-823, November 2007.

[2] S. Neusser and D. Grundler, "Magnonics: spin waves on the nanoscale”, Adv. Mater., vol.21, pp. 2927-2932, June 2009.

[3] T. Schneider, A.A. Serga, B. Leven, B. Hillebrands, R.L. Stamps, and M.P. Kostylev, "Realization of spin-wave logic gates", Appl. Phys. Lett., vol.92, 022505, January 2008.

[4] A.H. Lu, E.E. Salabas, and F. Schüth, "Magnetic nanoparticles: synthesis, protection, functionalization, and application", Angewandte Chemie International Edition, vol.46, pp. 1222-1244, February 2007.

[5] M. Sanles-Sobrido, M. Bañobre-López, V. Salgueiriño, M.A. Correa-Duarte, B. Rodríguez-González, J. Rivas, and L.M. Liz-Marzán, "Tailoring the magnetic properties of nickel nanoshells through controlled chemical growth", Journ. Mat. Chem., vol.20, pp. 73607365, July 2010.

[6] C.G. Hu, Y. Li, J.P. Liu, Y.Y. Zhang, G. Bao, B. Buchine, and Z.L. Wang, "Sonochemical synthesis of ferromagnetic core-shell $\mathrm{Fe}_{3} \mathrm{O}_{4}-\mathrm{FeP}$ nanoparticles and FeP nanoshells", Chem. Phys. Lett., vol.428, pp. 343-347, September 2006.

[7] M.N. Hansen, L.S. Chang, and A. Wei, "Resorcinarene-encapsulated gold nanorods: solvatochromatism and magnetic nanoshell formation", Supramolecular chemistry, vol.20, pp. 35-40, April 2008.

[8] Yu.I. Gorobets and V.V. Kulish, "Dipole-exchange spin excitations in a thin ferromagnetic nanoshell”, Central European Journal of Physics, No.11(3), pp. 375-379, March 2013. 